\title{
Analysis of car shredder polymer waste with Raman mapping and chemometrics
}

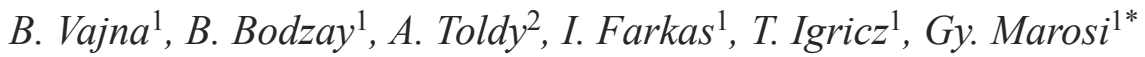 \\ ${ }^{1}$ Department of Organic Chemistry and Technology, Faculty of Chemical Technology and Biotechnology, Budapest \\ University of Technology and Economics, H-1111 Budapest, Budafoki út 8., Hungary \\ ${ }^{2}$ Department of Polymer Engineering, Faculty of Mechanical Engineering, Budapest University of Technology and \\ Economics, H-1111 Budapest, Müegyetem rkp. 3., Hungary
}

Received 15 June 2011; accepted in revised form 29 August 2011

\begin{abstract}
A novel evaluation method was developed for Raman microscopic quantitative characterization of polymer waste. Car shredder polymer waste was divided into different density fractions by magnetic density separation (MDS) technique, and each fraction was investigated by Raman mapping, which is capable of detecting the components being present even in low concentration. The only method available for evaluation of the mapping results was earlier to assign each pixel to a component visually and to count the number of different polymers on the Raman map. An automated method is proposed here for pixel classification, which helps to detect the different polymers present and enables rapid assignment of each pixel to the appropriate polymer. Six chemometric methods were tested to provide a basis for the pixel classification, among which multivariate curve resolution-alternating least squares (MCR-ALS) provided the best results. The MCR-ALS based pixel identification method was then used for the quantitative characterization of each waste density fraction, where it was found that the automated method yields accurate results in a very short time, as opposed to manual pixel counting method which may take hours of human work per dataset.
\end{abstract}

Keywords: recycling, micro-Raman, hyperspectral imaging, chemometrics, polymer waste

\section{Introduction}

Shredder wastes, even after thorough separation processes, consist of various components, the identification of which needs advanced analytical approach. The main components can be analysed by conventional spectroscopic methods, however, these provide only bulk information [1]. The quantitative determination of the composition and identification of the minor components destroying the processability and/or stability of the material needs additional efforts. It is of great importance to determine the sites where the degree of degradation is high and such components are present that induce mechanical and/or chemical deterioration. For these purposes the so-called hyperspectral chemical imaging techniques are promising extensions of the currently used methods.

Chemical imaging is a rapidly emerging analytical method gaining importance in multiple fields, such as food industry [2], pharmaceuticals [3-6], forensics [7] and polymers [8]. This group of techniques combines vibrational (mostly MIR, NIR or Raman) spectrometry with the spatial resolution of an optical system (usually a microscope). Either images at certain wavelengths are stacked together (global imaging), or distinct spectra are collected from a predetermined grid on the sample surface (point/line mapping), three-dimensional datasets are formed in a way that a vibrational spectrum corresponds to each point of the sample surface. Although the

\footnotetext{
${ }^{*}$ Corresponding author, e-mail: gmarosi@mail.bme.hu
}

(c) BME-PT 
terms 'mapping' and 'imaging' originally refer to different instrumental set-ups, their use is often considered interchangeable and 'imaging' is used as a general term to describe both approaches [3-5]. Similarly, in the present study, these terms are used as synonyms.

A large variety of questions can be answered using Raman chemical imaging, by utilizing the spatial information as well as the spectral signals. The miscibility of different binary polypropylene/polyurethane [8], polyethylene/polypropylene [9-11], polyamide/polytetrafluoroethylene [12] and ternary [13] polymer blends and their spatial structure can be studied using chemical imaging methods. Different types of heterogeneities (compositional, structural and morphological) can be defined and separately analysed [14]. Phase separations in polyfluorene have also been studied by Raman mapping [15], while other authors have investigated the effect of fillers on phase separation [16]. Material defects leading to deteriorated fatigue behaviour can be revealed [17], besides, surface characteristics and the effect of coating processes can also be monitored $[18,19]$. Due to the sharp and selective Raman peaks, crystallinity and solid state characteristics can be investigated with good efficiency [9, 20]. Raman chemical imaging of polymers is becoming especially popular in the field of pharmaceuticals [21, 22].

It has been proven that the evaluation of vibrational chemical images can be greatly enhanced with multivariate data analysis techniques. Several questions can be answered by such methods: component detection and identification, object classification, and quantitative determination of certain features (e.g. concentration of components).

When unknown samples are investigated, the spectra of the pure components may not always be available. However, the huge amount of data stored in the hyperspectral images make it possible to predict or estimate the pure component spectra and to determine the spatial distribution of components even when no or only limited a priori information is present about the samples. Sample-sample twodimensional correlation spectroscopy has been applied by Šašić et al. [23] to analyze multiple polymer blends. The visualization of polymer distributions was enhanced by principal component analy- sis (PCA) in the study of Stellman et al. [24] and by cosine correlation approach in the study of Morris et al. [19]. Multiple curve resolution methods have also been compared based on experiments with model samples in the fields of polymers [25] and pharmaceuticals [26, 27].

The analysis of waste materials is also an emerging issue where vibrational spectrometry and chemical imaging are very promising [28-34]. IR spectra, Raman spectra and hyperspectral images in the visible and NIR range enable the identification of postconsumer glasses [28], polymers [29], composts [30] and their contaminants $[29,30]$ via logical recognition rules [29] and multivariate image analysis [30]. On-line identification of polymer waste components can also be carried out with NIR imaging by using supervised classification methods if an appropriate training set (containing every polymer to be identified) is investigated previously $[32,33]$. These two NIR studies showed that a NIR image can be taken from the intact polymer waste and the different plastic objects can be immediately identified via chemometric spectrum classification. It has also been proven that chemical imaging is suitable for quantitative analysis based on the number of classified pixels in each class [34]. However, chemometric processing of vibrational chemical images have not yet been studied for quantitative analysis of polymers (in an earlier study carried out by Vajna $e t$ al. [34] the classification of Raman spectra was carried out manually, which is an extremely time-consuming procedure).

The aim of this study was to compare different chemometric methods in the quantification of different density fractions of car shredder polymer waste by Raman mapping. As waste materials often contain unknown substances, unsupervised classification and curve resolution techniques were tested that can be applied without using any kind of training sets or reference spectra. Since real-life samples were analyzed, the most prominent challenge in this case was the poor quality of the measured datasets (highly varying and often low signal-to-noise ratio, high fluorescent background and high number of outliers due to various other effects, such as detector saturation and the presence of dyes or other additives). 


\section{Materials and methods}

\subsection{Materials}

The analyzed car shredder polymer waste (CSPW) originated from a car shredder plant (Alcufer Ltd, Hungary), where cars and large household appliances are processed. At first, dust was removed with dry and wet processes, and then the magnetic metals were removed with a magnet, while the nonmagnetic metals were separated with a vortex separator. The remaining material, consisting of mainly polymers, was then separated to pre-defined density fractions by inverse magnetic density separation technique [35]. Four density fractions were separated for analysis, which are shown in Table 1.

Table 1. Acquired density fractions of car shredder polymer waste (CSPW) for Raman mapping analysis

\begin{tabular}{|c|l|}
\hline Density & Sample code \\
\hline$\rho<0.9 \mathrm{~g} / \mathrm{cm}^{3}$ & CSPW $<0.9$ \\
\hline $0.9 \mathrm{~g} / \mathrm{cm}^{3} \leq \rho<1 \mathrm{~g} / \mathrm{cm}^{3}$ & CSPW $0.9-1$ \\
\hline $1 \mathrm{~g} / \mathrm{cm}^{3} \leq \rho<1.05 \mathrm{~g} / \mathrm{cm}^{3}$ & CSPW $1-1.05$ \\
\hline $1.05 \mathrm{~g} / \mathrm{cm}^{3} \leq \rho<1.3 \mathrm{~g} / \mathrm{cm}^{3}$ & CSPW $1.05-1.3$ \\
\hline
\end{tabular}

\subsection{Raman mapping experiments}

Raman mapping spectra were collected using a LabRAM system (Horiba Jobin-Yvon, Lyon, France) coupled with an external $785 \mathrm{~nm}$ diode laser source (Sacher Lasertechnik, Marburg, Germany) and an Olympus BX-40 optical microscope (Olympus, Hamburg, Germany). Objectives of 10× magnification were used for optical imaging and spectrum acquisition. The laser beam is directed through the objective, and backscattered radiation is collected with the same objective. The collected radiation is directed through a notch filter that removes the Rayleigh photons, then through a confocal hole and the entrance slit onto a grating monochromator ( 950 groove $/ \mathrm{mm}$ ) that disperses the light before it reaches the CCD detector. The spectrograph was set to provide a spectral range of $550-1750 \mathrm{~cm}^{-1}$ and $3 \mathrm{~cm}^{-1}$ resolution.

The shredded polymer waste sample was ground in a liquid $\mathrm{N}_{2}$-cooled grinder to reduce the particle size to microscopic scale. The ground particles were pressed with a hydraulic press at 200 bar to provide $60 \mathrm{~mm} \times 60 \mathrm{~mm}$ flat surface for the Raman analysis. The measured area was $29 \times 29$ points in each case. Step size of $500 \mu \mathrm{m} \times 500 \mu \mathrm{m}$ was chosen between the adjacent points in order to minimize dependence between adjacent points. The spectrum acqui- sition time was $3 \mathrm{~s}$ per spectrum. 20 spectra were accumulated and averaged at each measured point (further also referred to as: 'pixel') to achieve acceptable signal-to-noise ratio.

\subsection{Data analysis}

Before chemometric evaluation, fluorescent background was removed using piece-wise linear baseline correction with manually chosen baseline points. The measured spectra were then normalized to unit area in order to eliminate the intensity deviation between the measured points (pixels). The Raman maps were then unfolded to a two-dimensional matrix form (X) of 841 rows (number of measured spectra in a map) and 1000 columns (number of wavenumber channels). A measured spectrum of the Raman map (a row in $\mathbf{X}$ ) can be considered as a vector in the 1000 dimensional (spectral) vector space.

K-means clustering was carried out with Statistica 9.0 software (StatSoft, USA). All other calculations described in Sections 2.3.1.- 2.3.6. were performed in MATLAB 7.6.0 (Mathworks, Natick, USA) with PLS_Toolbox 6.0.1 and MIA_Toolbox 2.0.1 (Eigenvector Research, Seattle, USA). The chemometric methods were tested on the Raman map of the CSPW 1.05-1.3 density fraction and the best one selected was used on all other fractions.

\subsubsection{Manual classification via visual inspection of spectra}

Reference classification was carried out manually by visual inspection of the spectra in the Raman chemical image. Each measured spectrum (further also referred to as 'object') was visually identified (using spectral library search when needed) and classified accordingly. Spectra containing no useful information were considered as unclassified (bad) spectra.

\subsubsection{Principal component analysis (PCA)}

PCA [36] is a factor-analysis based method that extracts the most important factors describing the information broadly distributed in a dataset. The data matrix $\mathbf{X}$ can be resolved into three matrices by performing singular value decomposition (Equation (1)):

$\mathbf{X}=\mathbf{U} \mathbf{\Lambda} \mathbf{V}^{\mathrm{T}}$ 
Theoretically, the first few loading vectors (first few rows in $\mathbf{V}^{\mathrm{T}}$ ) hold important spectral features, the others mainly consist of deviations and noise. The product of $\mathbf{U}$ and $\boldsymbol{\Lambda}$ provides the score matrix $\mathbf{T}$. These scores can be visualized in the principal component subspace (which is a subspace of the spectral vector space described in Section 2.3.) and allow visual separation of groups of objects. PCA was performed with the pca command of MATLAB PLS_Toolbox. In every case, the first 20 principal components were calculated.

\subsubsection{K-means clustering}

Clustering [37] is the most common algorithm in the family of unsupervised classification models. It is based on the fact that each object can be represented with a point in the spectral vector space, the position of which is described by the corresponding row vector in $\mathbf{X}$. If these points form groups in the vector space, these groups can be found by clustering algorithms.

$\mathrm{K}$-means clustering groups the objects into a given number of clusters (pre-determined by the user). Cluster sizes and positions are iteratively calculated in a way that within-cluster distances are minimized and between-cluster distances are maximized. Some elements may not be successfully included in any of the clusters.

Two types of initializations were tried for the calculations. In the first one (init 1), the distances among the initial cluster positions were maximized. In the second one (init2), initial cluster positions were chosen in a way that they would evenly span the spectral space (object distances were sorted and objects were taken at constant intervals). In each case, number of clusters was set to 20 and Euclidean distance was used for the iterations.

In order to improve the performance of clustering, the effect of an additional data preprocessing step (column standardization [38], i.e. subtracting the mean from each column and dividing each value with the standard deviation) was also tested.

\subsubsection{Classical least squares (CLS)}

Classical Least Squares method [38] uses the assumption of a bilinear model (Equation (2)):

$\mathbf{X}=\mathbf{C S}^{\mathrm{T}}+\mathbf{E}$
$\mathbf{S}^{\mathrm{T}}(k \cdot \lambda)$ is the set of reference (pure component) spectra, each spectrum consisting of $\lambda$ intensity values and forming a row in the matrix. $\mathbf{X}(p \cdot \lambda)$ is the matrix containing the mapping spectra, and $\mathbf{C}(p \cdot k)$ contains the vectors of spectral concentrations (each row in $\mathbf{C}$ contains the concentrations of the $k$ ingredients). The matrix $\mathbf{E}$ represents the residual noise. The alignment of the above mentioned matrices is visually illustrated in reference [4].

The spectral concentrations were estimated by Equation (3):

$\mathbf{C}=\mathbf{X S}\left(\mathbf{S}^{\mathrm{T}} \mathbf{S}\right)^{-1}$

Using CLS with all reference spectra of the expected polymers present, this method calculates the (spectral) concentration of each component (each possible polymer) in each pixel. As the particle sizes greatly exceeded the sampling volume during spectrum acquisition, in this case one measurement point was expected to correspond to only one polymer. Thus, if the calculated spectral concentration of a certain polymer reached a certain threshold level, the object was classified to the group of that particular polymer. Numerous threshold levels were tested to achieve the best results.

\subsubsection{Self-modelling mixture analysis (SMMA)}

SMMA [39] aims to find the purest variables (wavelength channels) by the statistical evaluation of the columns of the $\mathbf{X}$ matrix. The 'length' and 'purity' of these columns (i.e. wavenumbers) are determined based on the mean and standard deviation of the intensity values. After selecting the purest variables, the corresponding columns are used as a guess for the concentration matrix, and the pure component spectra are estimated by Equation (4).

$\mathbf{S}^{\mathrm{T}}=\left(\mathbf{C}^{\mathrm{T}} \mathbf{C}\right)^{-1} \mathbf{C}^{\mathrm{T}} \mathbf{X}$

The calculations were carried out with the 'Purity' option in PLS_Toolbox at a very high offset level '40'. (This corresponds to $\alpha=0.4$ ' (maximum intensity) offset value in the original SMMA method proposed by Windig and Guilment [39]).

\subsubsection{Multivariate curve resolution - alternating least squares (MCR-ALS)}

This method, as its name also implies, is an iterative approach with repeated, consecutive estimations of 
$\mathbf{C}$ from $\mathbf{S}^{\mathrm{T}}$, and vice versa [40]. Physical constraints can be applied between the steps, such as non-negativity of spectral concentrations and intensities, closure of concentration values (their sum is fixed to 1), normality of spectra, unimodality, etc. MCR-ALS needs an initial guess for either the concentrations or the spectra to start the iteration.

The iterations were performed with the $\mathrm{mcr}$ command of PLS_Toolbox, applying only non-negativity constraints (for both spectra and concentrations) and allowing 300 iteration steps. Two types of initial guesses were used: estimated spectra by SMMA without any offset, and the loadings calculated at offset '40' as described in Section 2.3.5.

\subsubsection{Positive matrix factorization (PMF)}

The algorithm originally developed by Paatero [41] aims to minimize the error (E) matrix of Equation (1) in a gradient-based manner. The errors can be weighted by the uncertainties at the different positions of the $\mathbf{X}$ matrix, but this feature was not used in this study since it was unnecessary with the experimental setup shown here.

PMF was carried out using the PMF-2 program. The default pseudorandom numbers were used as initial guesses for the pure spectra. The input error level $\left(\sigma_{\mathrm{ij}}\right)$ was set to 0.03 according to a thumb rule described in a guide to the PMF method [42].

\subsubsection{Simplex identification via split augmented lagrangian (SISAL)}

The method can be geometrically interpreted by finding the smallest simplex in the data space that encloses all measured spectra [43]. The apices of this simplex correspond to the pure component spectra, as all measured points are a mixture of these.

SISAL was carried out by the MATLAB implementation downloaded from the source given in the study of Lopes et al. [43]. Numerous different $\lambda$ values (which is signed $\tau$ in the MATLAB code) were tried to achieve the best resolution. Best results were achieved with $\lambda=1$ and 200 iteration steps.

\subsection{Data evaluation and visualization}

The estimated pure spectra (further also referred to as 'loadings') were visually compared to library reference spectra. The obtained scores or spectral concentrations of the meaningful loadings were refolded into a 2D array according to the spatial information in the original dataset. Object classification was carried out in the same way as described in Section 2.3.4. using the estimated spectral concentrations. This can be also considered as a 'binarization' method, i.e. the concentration of the most prominent component in a pixel is set to 1 and the concentration of the others is set to 0 . Visual classification maps were created as a multi-coloured overlaid image of these binarized concentration maps. This way, the different colours correspond to different polymers. Visualization of spectra and classification maps was carried out with LabSpec 5.41 (Horiba Jobin Yvon, Lyon, France).

Polymer composition in the different CSPW fractions was calculated by counting the number of objects in each class and dividing this sum by the total number of objects (841). This ratio was multiplied by 100 to give values in percentage.

Misclassification rate (MR) was determined for each chemometric method by comparing the class assignments between the actual method and the reference class assignments described in Section 2.3.1. $M R$ (in percentage) is calculated with Equation (5):

$\operatorname{MR}[\%]=\left(1-\frac{m}{841}\right) \cdot 100$

where $m$ is the number of matching classification assignments with the reference, i.e. the number of correct classifications in the Raman image; 841 is the total number of spectra in each dataset.

\section{Results and discussion}

The automated estimation of the composition of polymer waste is crucial when the question is whether a sample can be recycled. One aim in the present research was to determine whether the CSPW fractions of different density have a main component and what is the composition of these fractions. In our earlier reported Raman mapping results for quantitative polymer waste analyses the spectra in the Raman map were classified one-byone visually [34]. This was proven to be, however, an extremely time-consuming process, which is not acceptable for industrial or any other large scale application. The primary aim of the present study was to find the most appropriate chemometric 
method to identify the components present and simultaneously determine their concentrations in the sample.

The biggest challenge is the fact that these Raman datasets are of very poor quality due to numerous disturbing factors. As practically any polymers and rubbery materials can be present in the samples, which are also contaminated by other substances (e.g. glass, textile, wood), and each substance has its own response characteristics to the laser excitation, it is very difficult to find proper measurement conditions that work for most of the possible components present. Bad spectra can be obtained (1) if the peaks are blurred by high fluorescent background, (2) if the detector is saturated due to high fluorescence or unexpectedly intensive Raman bands, (3) due to the presence of dyes or other additives, (4) if a component suffers degradation during the spectrum acquisition.

A practiced expert equipped with appropriate spectral library can visually identify even bad quality spectra (if enough signal is present for the identification). The Raman images of each sample were evaluated visually in a way that the pixels containing the different polymers were visually counted. The most diverse density fraction, CSPW 1.05-1.3 was then evaluated in even more details: each pixel was classified visually. The efficiency of the studied, unsupervised chemometric methods was evaluated using the misclassification rate, i.e. what percentage of the pixels were (in)correctly classified. Then, the performance of the chemometric method with the lowest misclassification rate was further tested based on the quantitative analysis of all other density fractions compared to the reference composition determined visually.

\subsection{Visual classification of Raman spectra in the CSPW 1.05-1.3 Raman map}

Thorough visual inspection of the spectra of the CSPW 1.05-1.3 sample revealed the presence of nine polymers. Objects were grouped into the following nine classes: polystyrene (PS), polypropylene (PP), polycarbonate (PC), polyamide (PA), polyvinyl chloride (PVC), polyethylene terephtalate (PET), poly(methyl metacrylate) (PMMA), polyethylene (PE) and another unknown substance (unkn.) which could not be identified due to the fact that no matching spectrum was found in the spectral library.

\subsection{Exploratory analysis using PCA}

Principal component analysis is a very convenient tool to explore a dataset, determine the most prominent factors and to visualize the distribution of the objects in the spectral data space (more accurately, in its principal component subspace).

Figure 1 shows the score plot of the CSPW 1.05-1.3 dataset, proving that the objects indeed form distinct groups in the data space. It should be noted that the first and the fourth principal components (PC1 and PC4) were used for the score plot. PC2 and $\mathrm{PC} 3$, in spite of the fact that they explain more variance in the dataset, are not that discriminative of the different polymer classes. This is most likely due to the large number of outliers in the dataset,

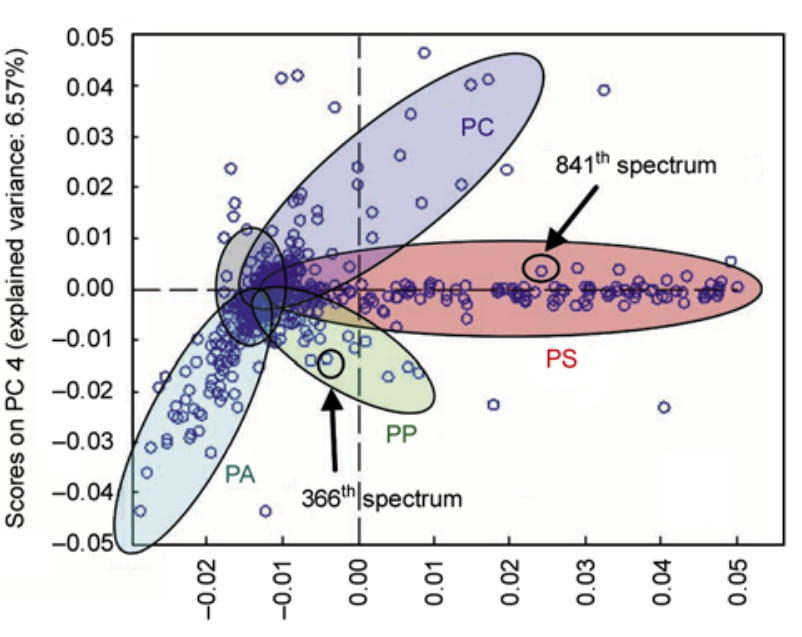

b)

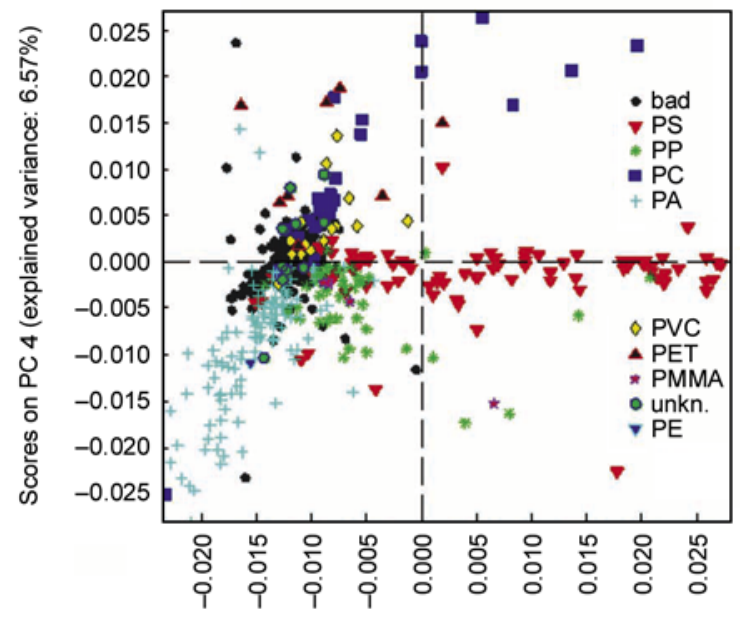

a)

Figure 1. PCA score plot of the CSPW 1.05-1.3 Raman map: a) with reference class assignments shown; b) without reference classes but with the prominent groups marked with an ellipse 
however, in chemical imaging it is always advised to visually check the principal components with lower eigenvalues (describing lower variances) as well [44].

It can be observed in Figure 1a that there is some overlap between the classes; however, the more prominent groups with a larger number of objects can be already distinguished (as also shown in Figure 1b). Even if we do not utilize the reference class information obtained visually, four polymers (PS, PP, PC and PA) can be identified by selecting certain objects in the most prominent groups (manually drawn ellipses in Figure 1b) and identifying these spectra. Further groups may be distinguished using other principal components for visualization. PCA is thus a convenient tool to get a general idea about the dataset. However, its main disadvantage is that PCA results can not be processed efficiently for unsupervised classification purposes (like those described in Section 2.4), i.e. the groups cannot be explicitly defined and the number of objects in these roughly identified groups cannot be determined. Therefore PCA cannot be used for quantitative evaluation. (It also has to be noted that not all data points are shown in Figure 1, both axes were truncated to give a clear view on all classes.)

\subsection{Estimation of pure component spectra}

Estimations for the spectra of the pure components present can be produced in a straightforward way using curve resolution methods (SMMA, MCRALS, PMF, SISAL), which provide 'loadings' that can be physically interpreted as spectra themselves. PCA also generates loadings, but these are always linear combinations of the real pure component spectra; thus, PCA gives worse estimations than curve resolution techniques [25-27]. Cluster analysis identifies groups among the objects in the dataset; the mean spectra of these clusters can be also considered as estimations for the pure component spectra (as similar spectra will most likely be placed in the same cluster).

The bad quality of the dataset reflects both in the mean spectra of the clusters and the calculated loadings (estimated pure component spectra) with the curve resolution techniques. Figure 2 shows the meaningful loadings calculated by MCR-ALS compared to the pure reference spectra of the identified polymers. Out of twenty loadings, only six are use- ful (L4 as PVC, L7 as PP, L12 as PC, L16 as PET, L17 as PS, L20 as PA). Loadings L5 and L9 are spectrally meaningful but practically not useful: L5 can be identified as the spectrum of a particular blue dye that often appears in the car shredder waste and is present in numerous polymers, and L9 holds similar information as L12 and corresponds to PET but with many peaks absent and lower signal-to-noise ratio. All other loadings correspond to artefacts due to baseline deviations and outliers due to detector saturation phenomena.

The findings above apply to all tested chemometric methods, i.e. the majority of the loadings (and the mean spectra of clusters) correspond to outliers and artefacts and a few are good estimations of the real spectra of the polymers present. Best results were achieved with MCR-ALS by resolving 6 polymer spectra out of the nine components present. It has to be noted, though, that L4 is a very poor estimation of the PVC spectrum as only one peak is present at $638 \mathrm{~cm}^{-1}$ instead of both peaks at 638 and $690 \mathrm{~cm}^{-1}$ (for comparison see Figure 2). The same 6 polymer spectra were resolved with PMF and SISAL. The

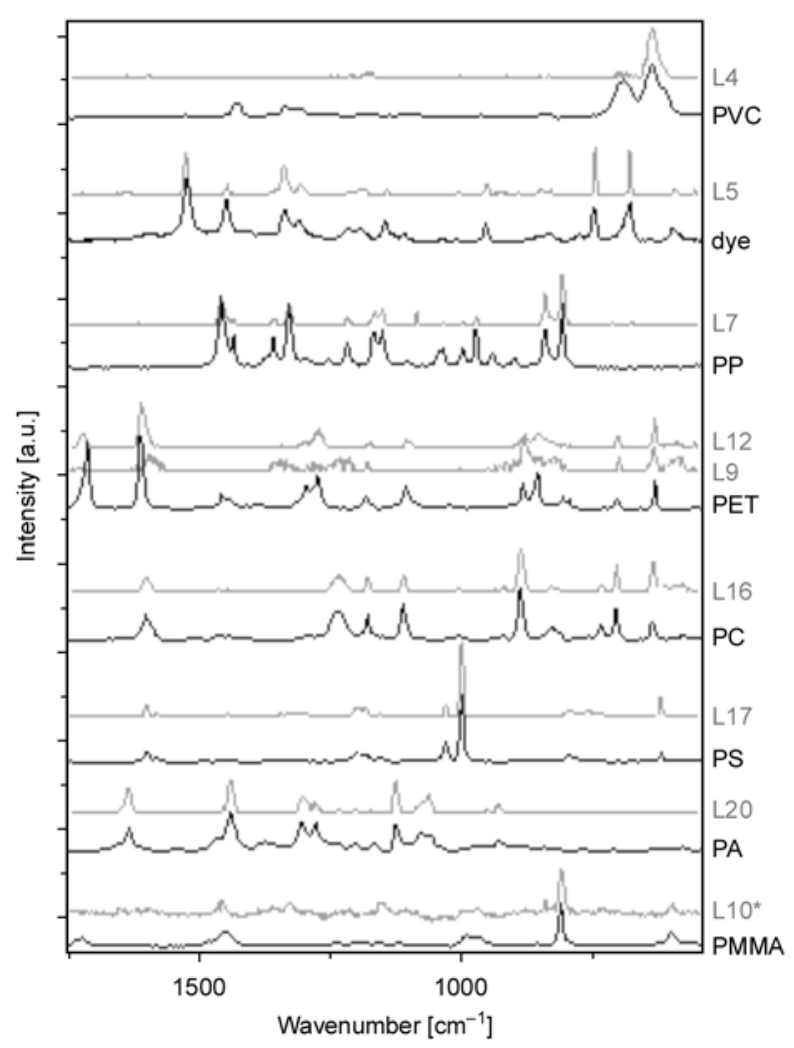

Figure 2. Selected MCR-ALS loadings (grey) of the CSPW 1.05-1.3 Raman map compared to reference spectra (black) of polymers and dyes. Loading 'L10', marked with an asterisk, was resolved with SMMA. 
presence of PVC in the dataset was not detected with SMMA, but PMMA spectrum was resolved instead, which was not found using the other curve resolution methods. K-means clustering showed worse performance than other techniques by only detecting five components (PS, PP, PC, PA and PET).

\subsection{Classification of measured points}

Section 3.3. proved that all studied methods (or their combinations) were feasible for qualitative analysis, since the spectra of the major components could be resolved from the dataset. However, the main question is whether correct quantitative analysis can be carried out as well.

As mentioned in Section 2.3.4., each measured point is expected to contain only one polymer. The basis of quantitative analysis in these cases is to calculate the number of points containing a particular polymer and dividing this number with the total number of measured points. Consequently, it is required that each pixel is classified, i.e. it is determined, which polymer it contains. This automated classification can then be compared to the reference assignments carried out visually, and the rate of misclassification (MR) can be calculated as a quantitative measure of the accuracy of these methods.

Automated classification is straightforward in the case of the K-means clustering method, where each point is included in one certain cluster: the only task is to assign all clusters to the appropriate polymers (or meaningless 'bad' points) based on the mean spectra of the clusters. However, this is not the case when curve resolution methods are applied: these methods provide 'scores' i.e. 'concentrations' for all components in every pixel, despite the assumption that every pixel corresponds to one polymer only. Therefore, another step has to be added which unambiguously assigns pixels to the components in the sample.
Pixel assignment to a particular polymer based on calculated concentrations can be carried out via two approaches. The most straightforward possibility would be to select the loading with the highest concentration and assign it to the pixel under evaluation. This approach, however, leads to high misclassification rate because of disturbances (fluorescence, detector cut-off or dye peaks) causing the highest score to be reached by non-meaningful loadings, even if the peaks of a polymer were also significantly present.

Another possibility to assign pixels is the following: if the Raman score of a certain polymer reaches a pre-defined threshold level, the pixel will be assigned to that particular polymer. This means that only those scores are considered which correspond to a loading already identified as a polymer spectrum. Since the polymer signals are mostly overlapped with disturbing phenomena and not with the signals of other polymers, this method provided unambiguous classification of almost all pixels. Where more than one polymer could be assigned to a pixel this way, the polymer with the higher score is to be considered. The score level $25 \%$ was defined as default level for thresholding (as such score is usually only observed when a polymer is significantly present), but numerous other threshold levels were also tested.

The best results obtained with each automated classification method are visually illustrated on Figure 3. As each polymer is shown with different colour, the real spatial distribution of polymers in the sample (determined by manual classification of spectra, Figure 3a) can be compared with the automated classification carried out with the chemometric methods. Black points correspond to pixels where no clear polymer signal was detected. Misclassification rate at various threshold levels are shown in Table 2.

Table 2. Misclassification rate with the different chemometric methods

\begin{tabular}{|c|c|c|c|c|c|c|c|c|c|c|c|}
\hline & & MCR-ALS & PMF & SMMA & SISAL & & & CLS & & & K-means \\
\hline \multirow{5}{*}{ 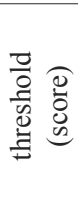 } & 0.30 & $16.1 \%$ & $18.4 \%$ & $24.7 \%$ & $44.7 \%$ & \multirow{5}{*}{ 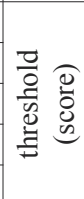 } & 2 & $53.6 \%$ & \multirow{5}{*}{ 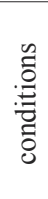 } & ${\text { init }{ }^{\mathrm{a}}}$ & $30.4 \%$ \\
\hline & 0.25 & $13.2 \%$ & $14.8 \%$ & $22.9 \%$ & $40.2 \%$ & & 1.5 & $44.1 \%$ & & init $2^{b}$ & $24.3 \%$ \\
\hline & 0.20 & $13.7 \%$ & $13.6 \%$ & $21.4 \%$ & $32.5 \%$ & & 1 & $44.6 \%$ & & & \multirow{3}{*}{$22.8 \%$} \\
\hline & 0.15 & $25.8 \%$ & $19.2 \%$ & $20.3 \%$ & $22.1 \%$ & & 1.5 & $51.6 \%$ & & standardization & \\
\hline & 0.10 & $44.2 \%$ & $33.7 \%$ & $24.9 \%$ & $54.9 \%$ & & 0.25 & $51.3 \%$ & & & \\
\hline
\end{tabular}

anitial cluster centres set with maximum possible initial distances within the data space.

b Initial cluster centres set at constant intervals in the data space. 

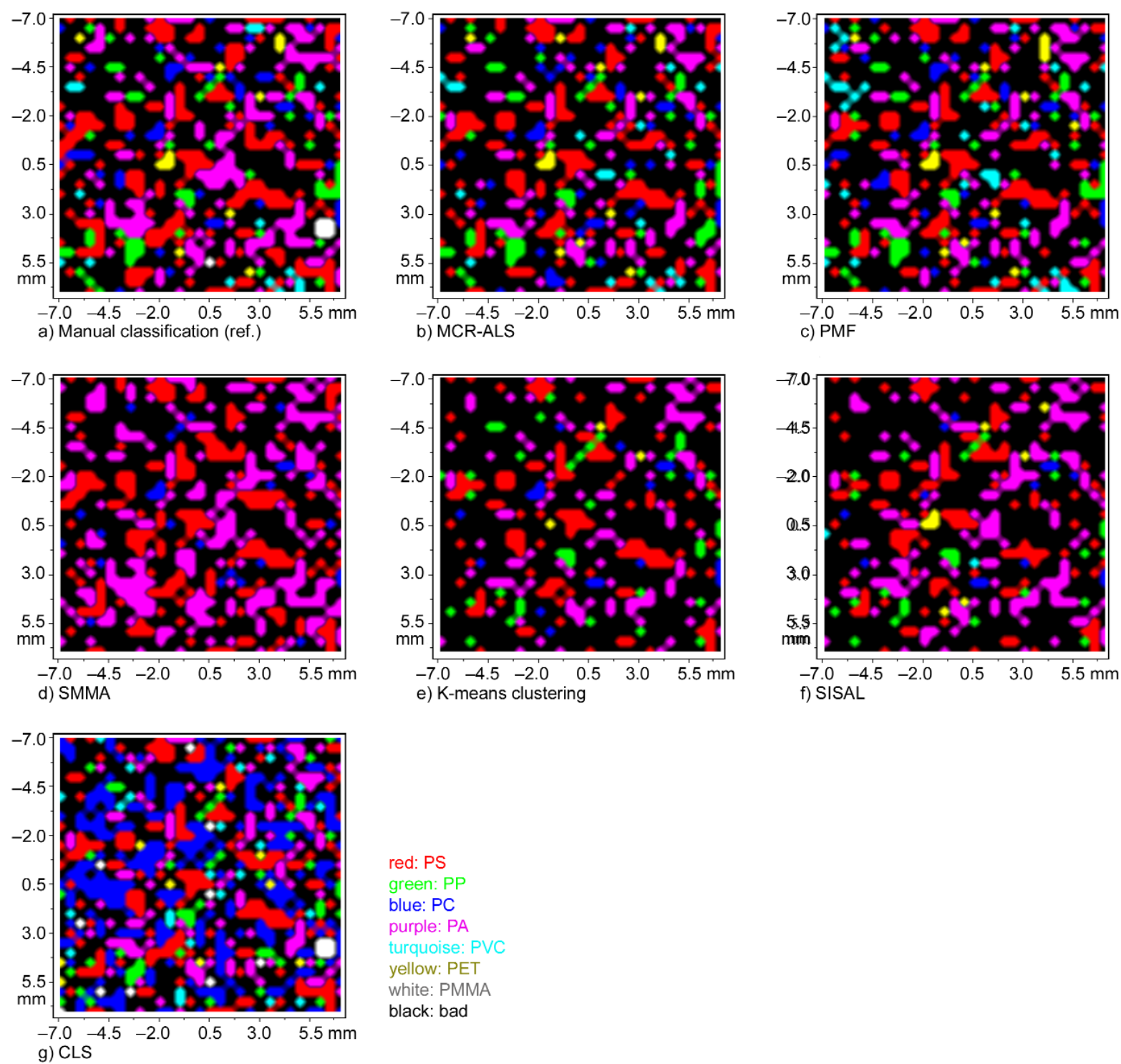

Figure 3. Pixel classification with different chemometric methods in comparison with the real polymer distribution in the CSPW 1.05-1.3 sample. (Threshold level 0.25 for curve resolution methods, K-means results in 'init2' mode and with column standardized dataset. For colour assignments the reader is referred to the web version of this article.)

Based on the scores obtained with MCR-ALS, the majority of pixels were correctly classified, especially those containing PS, PP or PET (red, green and yellow, respectively, in Figure 3b). MCR-ALS proved to be rather robust, as the outcome did not depend significantly on the applied threshold level (Table 2), making it suitable for the analysis of truly unknown substances (where the appropriate threshold level cannot be determined). The reason why the rate of misclassification increased when using very low threshold levels is that artefacts or other polymers with similar peaks can also achieve some score during the MCR-ALS resolution, but this applies to every other curve resolution method as well. Setting the threshold level too high results in the misclassification of pixels with good spectra (with unambiguous polymer signals) as bad pixels. Similar results were obtained with PMF with slightly worse performance mainly due to more frequent misclassification of bad spectra as PVC (light blue in Figure 3c). PMF also proved to be robust with small dependence of misclassification rate on the applied threshold level (Table 2).

SMMA scores allowed correct evaluation of PS and PA content as almost all of these pixels were correctly classified (Figure 3d). Moderately accurate results were obtained for PC. However, PP (green), PET (yellow) and PMMA (white) were not detected in the Raman map using any reasonable threshold level ( $10 \%$ or higher), even though their spectra 
were correctly resolved with the method. Further decrease of the detection threshold score leads to a high degree of misidentification of bad spectra as polymer signals. An advantage of SMMA would be that its overall misclassification rate does not depend much on the applied threshold level (Table 2), rendering this method fairly robust; however, it can only be used for the estimation of the major components.

The main advantage of K-means classification would be that bad spectra do not get misclassified as polymers. However, many pixels containing significant polymer signals were misclassified as bad spectra instead (large number of black points in Figure 3e). Misclassification rate could be significantly decreased by selecting the proper initialization method (designated init2 in Section 2.3.3.) and by applying column standardization on the dataset. Calculating larger number of clusters and applying other or further preprocessing steps did not increase the efficiency any further.

The performance of SISAL (Figure 3f) was the worst among the curve resolution methods most probably due to the fact that the algorithm cannot cope with such high number of outliers. Although fairly good results were achieved at $0.15 \%$ threshold level, even a slight change in the threshold resulted in strong deterioration of its efficiency. Thus, it cannot be expected to work well on a new unknown dataset where the threshold level cannot be optimized.

One can raise the question whether modelling the dataset with the pure component spectra using classical least squares method allows easy and straightforward evaluation of the dataset. The first, theoretical problem with CLS is that the scope of the present study is to successfully analyze and quantify unknown polymer waste samples, while CLS requires the knowledge of the components present in the sample. Even if a large number of pure spectra are (even if randomly) added to the calculations, one cannot know if all pure components have been included in the model. The bigger, practical problem with CLS is that even by adding the correct pure component spectra the rate of misclassification is very high. Figure $3 \mathrm{~g}$ shows that the misclassification of bad spectra is extremely frequent, in this case most of them misidentified as polycarbonate (blue on Figure 3g). Table 2 shows that neither using the default level, nor applying much higher thresh- old levels make this method feasible for the task, as bad pixels are frequently misclassified as one of the assumed polymers.

The explanation is that while curve resolution methods take the artefacts and noise effects into account by subsequent loadings, these effects cannot be taken into consideration with CLS. This also means that although these uninformative loadings resolved by curve resolution methods cannot be explicitly used and can be discarded in the evaluation, their role is very important in the correct prediction of the real components present in the waste sample.

\subsection{Estimation of CSPW composition with MCR-ALS}

Based on the findings in Section 3.4., the most efficient method to properly identify a component in a pixel is MCR-ALS. In the present section, this method is used for the estimation of the composition in the case of each CSPW density fraction. For classification purposes, the default threshold level of $25 \%$ was used. Real composition was calculated by visually counting the number of spectra in each CSPW Raman map to provide a reference for the MCR-ALS calculations.

Results for all the fractions of CSPW are shown in Table 3. MCR-ALS provided approximately the same results as the manual pixel counting (reference) method, while a tremendous amount of time can be saved. Major deviations from the reference were observed only in a few cases. It can be generally stated that the magnitude of error seen in Table 3 is well within tolerable limits. This makes the combination of Raman microscopic mapping and chemometrics a reliable automatic method for the quantitative characterization of polymer waste samples. Recyclability of wastes depends both on their major constituents and traces of contaminants. While the major components with high mass fractions may be identified using non-imaging spectroscopic methods as well, the advantage of Raman microscopic mapping over conventional bulk spectroscopic methods is the complementary detection and quantification of minor components and degraded polymers that affect the processability of the waste fraction. For example, the most prominent component in CSPW fraction 1-1.05 is polystyrene, however, it contains a significant amount of PVC and PET 
Table 3. Comparison of quantitative results obtained with the MCR-ALS based pixel classification and the reference method

\begin{tabular}{|c|c|c|c|c|c|c|c|c|c|c|c|c|}
\hline \multirow{2}{*}{$\begin{array}{l}\text { Density } \\
\mathrm{g}^{\mathrm{g}} \mathrm{cm}^{3}\end{array}$} & \multirow[t]{2}{*}{ Method } & \multicolumn{11}{|c|}{ Percentage of pixels containing the polymer [\%] } \\
\hline & & $\mathbf{P P}$ & degr. PP & $\mathbf{P E}$ & degr. $P E$ & PS & PVC & $\mathbf{P C}$ & PM-MA & PET & PA & unk. \\
\hline \multirow{2}{*}{$0-0.9$} & MCR & 54.0 & 38.6 & 7.4 & - & - & - & - & - & - & - & - \\
\hline & ref. & 57.9 & 41.4 & 0.4 & - & 0.2 & - & - & - & - & 0.2 & - \\
\hline \multirow{2}{*}{$0.9-1$} & MCR & 20.3 & 2.3 & 5.9 & 16.7 & 54.8 & - & - & - & - & - & - \\
\hline & ref. & 21.4 & 4.7 & 4.7 & 16.7 & 52.5 & - & - & - & - & - & - \\
\hline \multirow{2}{*}{$1-1.05$} & MCR & 7.8 & 0.5 & - & - & 78.7 & 10.1 & - & - & 1.8 & 1.1 & - \\
\hline & ref. & 11.7 & 0.4 & - & - & 84.4 & 1.2 & 0.4 & - & 0.2 & 1.4 & 0.2 \\
\hline \multirow{2}{*}{$1.05-1.3$} & MCR & 13.2 & - & - & - & 40.8 & 5.1 & 11.8 & - & 3.2 & 25.9 & - \\
\hline & ref. & 10.6 & - & 0.2 & - & 39.2 & 3.3 & 10.2 & 1.1 & 2.4 & 30.8 & 2.2 \\
\hline
\end{tabular}

which limit its recyclability. In contrast, density fractions below $0.9 \mathrm{~g} / \mathrm{cm}^{3}$ only contain PE and PP, hence were proven to be well recyclable. Additionally, it can be seen in the low density fractions that a significant amount of the polymer is degraded to some extent (spectra of intact and degraded polymers were compared in details in the study of Vajna et al. [34]), which also contains some information about the expectable quality of the recycled product.

\section{Conclusions}

Car shredder polymer waste was separated to different density fractions and the resulting samples were investigated with Raman mapping. A novel pixel identification method was developed based on an appropriate chemometric algorithm for time-efficient and accurate evaluation of the Raman maps. These datasets posed serious challenges due to tremendous amount of noise, outliers and measurement/preprocessing artefacts present in the data.

Using an appropriately diverse density fraction, the efficiency of six chemometric methods was compared to one another and to the reference visual evaluation. MCR-ALS was found to be the most robust method achieving the smallest misclassification rate, i.e. the highest accuracy. This method was then tested in the quantitative characterization of all density fractions of two polymer waste batch samples. The results proved that appropriate quantification can be carried out with MCR-ALS, also revealing the presence and estimating the amount of trace polymers and degraded parts which may influence the recyclability of the sample or the quality of the future product. While visual evaluation and manual pixel counting of a Raman map requires hours to perform, MCR-ALS calculations and subsequent pixel identification requires much less human work and reduces the time spent to minutes.

Based on the results shown in the present study, the combination of Raman mapping and appropriate chemometrics can greatly enhance the polymer recycling technologies by detailed characterization and quantitative determination of polymer waste samples. As the method developed here is based on an unsupervised curve resolution method, the investigations do not require any prior information about the samples; thus, completely unknown polymer samples can also be characterized.

\section{Acknowledgements}

The research was supported by the OTKA Research Fund (code K76346), ERA Chemistry (code NN 82426), W2Plastics EU7 Project (code 212782), and the Hungarian project TECH_08-A4/2-2008-0142. This work is connected to the scientific program of the 'Development of quality-oriented and harmonized $\mathrm{R}+\mathrm{D}+\mathrm{I}$ strategy and functional model at BME' project. This project is supported by the New Széchenyi Plan (Project ID: TÁMOP-4.2.1/B-09/1/KMR2010-0002).

The authors express their thanks to the University of Miskolc, Hungary for the magnetic density separation of car shredder polymer waste samples. Pentti Paatero is thanked for the useful advice on the PMF program. The first author would also like to express his thanks to Péter Egyedi, László Hortobágyi, Tamás Pálinkás, Dániel Sándor, István Győrfi and Csaba Bokros (IHM) for sharing their views and inner thoughts.

\section{References}

[1] Pereira A. G. B., Paulino A. T., Rubira A. F., Muniz E. C.: Polymer-polymer miscibility in $\mathrm{PEO} /$ cationic starch and PEO/hydrophobic starch blends. Express Polymer Letters, 4, 488-499 (2010).

DOI: $10.3144 /$ expresspolymlett.2010.62 
[2] Gowen A. A., Taghizadeh M., O’Donnell C. P.: Identification of mushrooms subjected to freeze damage using hyperspectral imaging. Journal of Food Engineering, 93, 7-12 (2009).

DOI: $10.1016 /$ j.jfoodeng.2008.12.021

[3] Gowen A. A., O’Donnell C. P., Cullen P. J., Bell S. E. J.: Recent applications of chemical imaging to pharmaceutical process monitoring and quality control. European Journal of Pharmaceutics and Biopharmaceutics, 69, 10-22 (2008).

DOI: $10.1016 /$ j.ejpb.2007.10.013

[4] Gendrin C., Roggo Y., Collet C.: Pharmaceutical applications of vibrational chemical imaging and chemometrics: A review. Journal of Pharmaceutical and Biomedical Analysis, 48, 533-553 (2008).

DOI: 10.1016/j.jpba.2008.08.014

[5] Amigo J. M.: Practical issues of hyperspectral imaging analysis of solid dosage forms. Analytical and Bioanalytical Chemistry, 398, 93-109 (2010).

DOI: $10.1007 / \mathrm{s} 00216-010-3828-\mathrm{Z}$

[6] Gordon K. C., McGoverin C. M.: Raman mapping of pharmaceuticals. International Journal of Pharmaceutics, 417, 151-162 (2010).

DOI: $10.1016 /$ j.ijpharm.2010.12.030

[7] Widjaja E., Garland M.: Use of Raman microscopy and band-target entropy minimization analysis to identify dyes in a commercial stamp. Implications for authentication and counterfeit detection. Analytical Chemistry, 80, 729-733 (2008).

DOI: $10.1021 /$ ac701940k

[8] Schaeberle M. D., Karakatsanis C. G., Lau C. J., Treado P. J.: Raman chemical imaging: Noninvasive visualization of polymer blend architecture. Analytical Chemistry, 67, 4316-4321 (1995).

DOI: $10.1021 / \mathrm{ac} 00119 \mathrm{a} 018$

[9] Furukawa T., Sato H., Kita Y., Matsukawa K., Yamaguchi H., Ochiai S., Siesler H. W., Ozaki Y.: Molecular structure, crystallinity and morphology of polyethylene/polypropylene blends studied by Raman mapping, scanning electron microscopy, wide angle X-ray diffraction, and differential scanning calorimetry. Polymer Journal, 38, 1127-1136 (2006).

DOI: 10.1295/polymj.PJ2006056

[10] Quintana S. L., Schmidt P., Dybal J., Kratochvíl J., Pastor J. M., Merino J. C.: Microdomain structure and chain orientation in polypropylene/polyethylene blends investigated by micro-Raman confocal imaging spectroscopy. Polymer, 43, 5187-5195 (2002).

DOI: 10.1016/S0032-3861(02)00384-1

[11] Schmidt P., Dybal J., Ščudla J., Raab M., Kratochvíl J., Eichhorn K-J., Quintana S. L., Pastor J. M.: Structure of polypropylene/polyethylene blends assessed by polarised PA-FTIR spectroscopy, polarised FT Raman spectroscopy and confocal Raman microscopy. Macromolecular Symposia, 184, 107-122 (2002). DOI: $10.1002 / 1521-3900(200208) 184: 1<107:: A I D-$ MASY107>3.0.CO;2-X
[12] Gupper A., Wilhelm P., Schmied M., Ingolic E.: Morphology of a PA/PTFE blend studied by Raman imaging. Macromolecular Symposia, 184, 275-285 (2002). DOI: 10.1002/1521-3900(200208)184:1<275::AIDMASY275>3.0.CO;2-9

[13] Markwort L., Kip B.: Micro-Raman imaging of heterogeneous polymer systems: General applications and limitations. Journal of Applied Polymer Science, 61, 231-254 (1996).

DOI: 10.1002/(SICI)1097-4628(19960711)61:2<231:: AID-APP6>3.0.CO;2-Q

[14] Ellis G.: Studies on the heterogeneity of polymeric systems by vibrational microscopy. Macromolecular Symposia, 184, 37-47 (2002).

DOI: $10.1002 / 1521-3900(200208) 184: 1<37:: A I D-$ MASY37>3.0.CO;2-4

[15] Stevenson R., Arias A. C., Ramsdale C., MacKenzie J. D., Richards D.: Raman microscopy determination of phase composition in polyfluorene composites. Applied Physics Letters, 79, 2178-2180 (2001).

DOI: $\underline{10.1063 / 1.1407863}$

[16] Appel R., Zerda T. W., Waddell W. H.: Raman microimaging of polymer blends. Applied Spectroscopy, 54, 1559-1566 (2000).

DOI: $\underline{10.1366 / 0003702001948808}$

[17] Marissen R., Schudy D., Kemp A. V. J. M., Coolen S. M. H., Duijzings W. G., Van Der Pol A., Van Gulick A. J.: The effect of material defects on the fatigue behaviour and the fracture strain of ABS. Journal of Materials Science, 36, 4167-4180 (2001).

DOI: 10.1023/A:1017960704248

[18] Morris H. R., Munroe B., Ryntz R. A., Treado P. J.: Fluorescence and Raman chemical imaging of thermoplastic olefin (TPO) adhesion promotion. Langmuir, 14, 2426-2434 (1998). DOI: $10.1021 / \mathrm{la} 971122 \mathrm{~g}$

[19] Morris H. R., Turner J. F. II, Munro B., Ryntz R. A., Treado P. J.: Chemical imaging of thermoplastic olefin (TPO) surface architecture. Langmuir, 15, 2961-2972 (1999). DOI: $10.1021 / \mathrm{la} 980653 \mathrm{~h}$

[20] Ellis G., Gómez M., Marco C.: Practical considerations in the study of main-chain thermotropic liquidcrystalline polymers by vibrational microscopy. Analusis, 28, 22-29 (2000). DOI: 10.1051/analusis:2000280022

[21] Nagy Zs. K., Nyúl K., Wagner I., Molnár K., Marosi Gy.: Electrospun water soluble polymer mat for ultrafast release of donepezil HCl. Express Polymer Letters, 4, 763-772 (2010).

DOI: $10.3144 /$ expresspolymlett.2010.92

[22] Patyi G., Bódis A., Antal I., Vajna B., Nagy Zs., Marosi Gy.: Thermal and spectroscopic analysis of inclusion complex of spironolactone prepared by evaporation and hot melt methods. Journal of Thermal Analysis and Calorimetry, 102, 349-355 (2010). DOI: $10.1007 / \mathrm{s} 10973-010-0936-0$ 
[23] Šašić S., Jiang J-H., Sato H., Ozaki Y.: Analyzing Raman images of polymer blends by sample-sample two-dimensional correlation spectroscopy. Analyst, 128, 1097-1103 (2003).

DOI: $10.1039 / B 303245 \mathrm{~K}$

[24] Stellman C. M., Booksh K. S., Muroski A. R., Nelson M. P., Myrick M. L.: Principal component mapping applied to Raman microspectroscopy of fiber-reinforced polymer composites. Science and Engineering of Composite Materials, 7, 51-80 (1998).

DOI: 10.1515/SECM.1998.7.1-2.51

[25] Duponchel L., Elmi-Rayaleh W., Ruckebusch C., Huvenne J. P.: Multivariate curve resolution methods in imaging spectroscopy: Influence of extraction methods and instrumental perturbations. Journal of Chemical Information and Modeling, 43, 2057-2067 (2003). DOI: $10.1021 / \mathrm{ci034097 \textrm {V }}$

[26] Gendrin C., Roggo Y., Collet C.: Self-modelling curve resolution of near infrared imaging data. Journal of Near Infrared Spectroscopy, 16, 151-157 (2008). DOI: $10.1255 /$ jnirs. 773

[27] Vajna B., Patyi G., Nagy Zs., Bódis A., Farkas A., Marosi Gy.: Comparison of chemometric methods in the analysis of pharmaceuticals with hyperspectral Raman imaging. Journal of Raman Spectroscopy, 42, 1977-1986 (2011). DOI: $10.1002 /$ jrs. 2943

[28] Farcomeni A., Serranti S., Bonifazi G.: Non-parametric analysis of infrared spectra for recognition of glass and glass ceramic fragments in recycling plants. Waste Management, 28, 557-564 (2008).

DOI: $10.1016 /$ j.wasman.2007.01.019

[29] Serranti S., Gargiulo A., Bonifazi G.: The utilization of hyperspectral imaging for impurities detection in secondary plastics. The Open Waste Management Journal, 3, 56-70 (2010).

DOI: $10.2174 / 18764002301003010056$

[30] Bonifazi G., Serranti S., Bonoli A., Dall'Ara A.: Innovative recognition-sorting procedures applied to solid waste: The hyperspectral approach. in: 'Sustainable development and planning IV, Vol. 2' (eds.: Brebbia C. A., Neophytou M., Beriatos E., Ioannou I., Kungolos A. G.), WIT Press, Southampton, 885-894 (2009). DOI: $10.2495 /$ SDP090832

[31] Serranti S., Bonifazi G.: Hyperspectral imaging based recognition procedures in particulate solid waste recycling. World Review of Science, Technology and Sustainable Development, 7, 271-281 (2010). DOI: $10.1504 / W R S T S D .2010 .032529$
[32] Kulcke A., Gurschler C., Spöck G., Leitner R., Kraft M.: On-line classification of synthetic polymers using near infrared spectral imaging. Journal of Near Infrared Spectroscopy, 11, 71-81 (2003).

DOI: $10.1255 /$ jnirs. 355

[33] Leitner R., Mairer H., Kercek A.: Real-time classification of polymers with NIR spectral imaging and blob analysis. Real-Time Imaging, 9, 245-251 (2003). DOI: $10.1016 /$ j.rti.2003.09.016

[34] Vajna B., Palásti K., Bodzay B., Toldy A., Patachia S., Buican R., Catalin C., Tierean M.: Complex analysis of car shredder light fraction. The Open Waste Management Journal, 3, 47-56 (2010).

DOI: $10.2174 / 18764002301003010046$

[35] Bakker E. J., Rem P. C., Fraunholcz N.: Upgrading mixed polyolefin waste with magnetic density separation. Waste Management, 29, 1712-1717 (2009). DOI: 10.1016/j.wasman.2008.11.006

[36] Malinowski E. R.: Factor analysis in chemistry. Wiley, New York (2004).

[37] Hastie T., Tibshirani R., Friedman J.: The elements of statistical learning: Data mining, inference, and prediction. Springer, New York (2009).

[38] Mark H., Workman J.: Chemometrics in spectroscopy. Academic Press, Amsterdam (2007).

[39] Windig W., Guilment J.: Interactive self-modeling mixture analysis. Analytical Chemistry, 63, 14251432 (1991). DOI: $10.1021 / \mathrm{ac} 00014 \mathrm{a} 016$

[40] Tauler R.: Multivariate curve resolution applied to second order data. Chemometrics and Intelligent Laboratory Systems, 30, 133-146 (1995).

DOI: $10.1016 / 0169-7439(95) 00047-X$

[41] Paatero P.: Least squares formulation of robust nonnegative factor analysis. Chemometrics and Intelligent Laboratory Systems, 37, 23-35 (1997). DOI: $10.1016 / \mathrm{S} 0169-7439(96) 00044-5$

[42] Paatero P.: The multilinear engine - A table-driven, least squares program for solving multilinear problems, including the $n$-way parallel factor analysis model. Journal of Computational and Graphical Statistics, 8, 854-888 (1999). DOI: $10.2307 / 1390831$

[43] Lopes M. B., Wolff J-C., Bioucas-Dias J. M., Figueiredo M. A. T.: Near-infrared hyperspectral unmixing based on a minimum volume criterion for fast and accurate chemometric characterization of counterfeit tablets. Analytical Chemistry, 82, 1462-1469 (2010). DOI: $10.1021 /$ ac902569e

[44] Šašić S.: An in-depth analysis of Raman and nearinfrared chemical images of common pharmaceutical tablets. Applied Spectroscopy, 61, 239-250 (2007). DOI: $10.1366 / 000370207780220769$ 